Crise(s) dans le monde ibérique et ibéro-américain

\title{
Vertiges de soi : Crises de l'écriture du moi dans le roman mexicain contemporain
}

\section{Véronique Pitois Pallares}

\section{(2) OpenEdition}

1 Journals

\section{Édition électronique}

URL : https://journals.openedition.org/cher/3564

DOI : $10.4000 /$ cher.3564

ISSN : 2803-5992

Éditeur

Presses universitaires de Strasbourg

\section{Édition imprimée}

Date de publication : 1 décembre 2015

Pagination : 85-92

ISBN : 978-2-86820-913-9

ISSN : 1968-035X

\section{Référence électronique}

Véronique Pitois Pallares, «Vertiges de soi : Crises de l'écriture du moi dans le roman mexicain contemporain », reCHERches [En ligne], 15 | 2015, mis en ligne le 01 décembre 2021, consulté le 20 décembre 2021. URL : http://journals.openedition.org/cher/3564; DOI : https://doi.org/10.4000/cher. 3564

\section{cc) (i) (ㅇ)}

Ce(tte) œuvre est mise à disposition selon les termes de la Licence Creative Commons Attribution -

Pas d'Utilisation Commerciale - Partage dans les Mêmes Conditions 4.0 International. 


\title{
Vertiges de soi : \\ Crises de l'écriture du moi dans le roman mexicain contemporain
}

\author{
Véronique Pitois Pallares \\ Université Paul Valéry - Montpellier 3
}

\begin{abstract}
$\mathrm{L}$ e roman mexicain du début de ce siècle s'inscrit dans un double contexte. Tout d'abord celui de la crise du sujet, l'un des versants de la crise de la modernité (Touraine 1992), et de la mort de l'auteur, ou en tout cas de l'autorité auctoriale, proclamée par Barthes en 1967. Les métarécits ou grands récits lyotardiens entrainent dans leur chute le récit tout court et la possibilité d'accéder à la vérité; le scepticisme s'empare des consciences et donne lieu à ce que Sarraute a désigné comme l'ère du soupçon (Sarraute 1956). Dès lors, dans le domaine des écritures du moi, on doute systématiquement de la véracité des données référentielles telles que l'identité de l'auteur et du narrateur autobiographe ou l'authenticité des souvenirs, mais on doute aussi, paradoxalement, de la fictionnalité des romans, soupçonnés à leur tour de s'alimenter de la vie de l'écrivain. Ainsi Robbe-Grillet affirme-t-il, au seuil de son premier livre à prétention ouvertement autobiographique: «Je n’ai jamais parlé d’autre chose que de moi. Comme cétait de l'intérieur, on ne s'en est guère aperçu.» (Robbe-Grillet 1984: 10). On remet donc autant en question la viabilité de l'écriture de soi que la possibilité de ne pas sécrire, soi, dans un écrit prétendument égo-décentré.

Lautre contexte dans lequel émergent les romans mexicains de ces quinze ou vingt dernières années est celui, bien connu lui aussi, du post-boom. La production des auteurs du fameux boom littéraire latino-américain des années 1960-1970 est marquée notamment par le réalisme magique et le réel merveilleux, et par l'ambition du roman total, énoncée par Vargas Llosa et dont l'archétype est la création de l'univers macondien de Cent ans de solitude (Vargas Llosa 2007: 9). Naturellement, les générations postérieures se sont démarquées des auteurs du boom et des esthétiques qui lui ont été associées. Depuis la fin
\end{abstract}

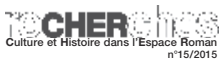


des années soixante-dix, on a ainsi assisté à un renouveau des genres référentiels comme le témoignage et la chronique, mis à l'honneur notamment par l'écrivaine et journaliste mexicaine Elena Poniatowska, et par Carlos Monsiváis ou Jorge Ibargüengoitia, respectivement. Dans la même veine de récupération des voix mineures et de l'histoire non officielle ou périphérique, Seymour Menton (1993) observe l'apparition du nouveau roman historique latino-américain qui compte parmi ses représentants Jorge Ibargüengoitia ou Rosa Beltrán. On constate aussi un nouvel engouement pour le roman policier, sous la forme du polar, noir et grinçant à souhait, parfaitement à même de représenter la violence des sociétés latino-américaines; les œuvres de Paco Ignacio Taibo II ou Elmer Mendoza séduisent ainsi un large éventail de lecteurs. Au Mexique toujours, à la fin des années soixante, la littérature de la Onda se pose en exemple de la contreculture et fait la place belle à l'argot et aux préoccupations des jeunes de la classe moyenne du centre du pays. Plus récemment, on observe chez de nombreux auteurs un mouvement de contre-contre-culture, c'est-à-dire un retour à des préoccupations esthétiques assumées, en même temps qu'une ambition universalisante. Ces préoccupations esthétiques, dans la pratique, conduisent jusqu'à une voie expérimentale, dans les œuvres d’auteurs tels que Mario Bellatin, Cristina Rivera Garza ou Jorge Volpi.

L'une des thématiques de prédilection de cette génération est l'identité subjective, le cheminement introspectif, dont le modèle canonique à battre en brèche est celui de l'autobiographie traditionnelle. Genre référentiel s'il en est, l'autobiographie a pour socle, selon Philippe Lejeune (1975), l'identité entre le je qui écrit (l'auteur), le je qui raconte (le narrateur) et le je ou le moi raconté (le personnage).

Les éléments d'émancipation par rapport au modèle du genre touchent chacun de ces piliers: l'identité auteur-narrateur-personnage, lécriture de soi dans sa linéarité et sa lisibilité, et la cohérence du moi couché sur le papier. D’autre part, après l'avalanche de héros nationaux qui a déferlé sur la littérature latino-américaine depuis le XIx siècle, au gré des mouvements délaboration patriotique et de la recherche exaltée de l'identité nationale, on voit se multiplier depuis la seconde moitié du $\mathrm{Xx}^{\mathrm{e}}$ siècle les antihéros, les personnages marginaux en proie au doute ou les personnages dysfonctionnels et leurs démons intérieurs. Ce motif a été fortement influencé par l'individualisme qui a suivi la fin des utopies collectives modernes. On retrouve alors un certain nombre de narrateurs psychotiques ou menacés par le spectre de la folie. La cohérence même du moi portraituré est problématisée dans des écrits autobiographiques, autofictionnels, ou dans des fictions homodiégétiques publiées au Mexique au cours des toutes récentes années 2000 .

On pensera par exemple à la narratrice du roman El huésped de Guadalupe Nettel, qui tente de lutter tant bien que mal contre l'invasion du parasite qu'elle porte en elle et qui colonise peu à peu sa conscience, ses yeux et ses actes:

Sabía que dentro de mí también vivía una cosa sin forma imaginable que jugaba cuando yo jugaba, comía cuando yo comía, era niña mientras yo lo era. Estaba 
segura de que algún día La Cosa iba a manifestarse, a dar signos de vida y, aunque la idea me parecía espeluznante, no dejaba de buscar esos signos en todos los pasillos de mi vida cotidiana como otras personas rastrean las espinillas que hay sobre su cara o las costras de grasa debajo del cabello. Cualquier cambio inexplicable en mi estado de ánimo, cualquier exabrupto, significaba una posible señal. Era muy poco lo que sabía en aquel tiempo de ese huésped interno. Sabía que su respiración era semejante a un pulpo, cuyos tentáculos pegajosos desplegaba por la noche a lo largo de mi cuarto; sabía que nada le resultaba tan hiriente como la luz y que, si alguna vez llegaba a dominarme, me condenaría a la oscuridad más absoluta; sabía en pocas palabras que era mi peor enemiga.

(Nettel 2006: 13-14)

Cette chose, La Cosa, est tour à tour présentée comme un alien envahisseur surgi du tréfonds de la jeune fille elle-même, ou comme le revers ténébreux d'une personnalité dont la réversibilité ou la mutabilité est suggérée dès le prénom palindrome: Ana. Dans un récit de facture somme toute assez classique, la tension narrative repose sur l'absorption progressive d'Ana par son hôte et sur la métamorphose qui la mène de la lumière de la ville de Mexico vers la cécité et les ténèbres du métro. Cet infra-monde métropolitain est présenté selon le motif classique du mundus inversus, comme pour insister sur la notion d'envers et d'endroit qui caractérise à la fois le personnage, Ana, l'espace dans lequel elle évolue et léchelle des valeurs sociales qui sont les siennes.

C'est aussi le cas de El camino de Santiago, seul roman à ce jour de Patricia Laurent Kullick, dans lequel la narratrice a bien du mal à faire cohabiter ses deux personnalités, Mina, jeune écervelée hédoniste et inconsciente, et Santiago, la voix mâle de la raison qui craint que les décisions dangereuses de Mina causent leur perte physique et mentale:

Los otros miedos, indescifrables como relámpagos que ciernen la sangre, son de Santiago, el intruso que invadió mi cuerpo cuando abrí la primera vena. [...]

Antes de hallar asilo en el torrente sanguíneo, Santiago me rondaba. Invisible soplaba su aliento sobre mi hombro. Me acechaba como la antítesis del ángel guardián, esperando el gran momento de flaqueza para integrar su perdida dimensión en la mía. Mientras trazaba la topografía de las rutas encefálicas que lo albergan, su proximidad me dispersaba obligándome a traficar cual si robara cada memoria de los primeros años, cuando Mina y yo penetrábamos reglas y límites humanos con el entusiasmo de un colibrí. (Laurent Kullick 2003: 7)

Cette scission, que l’on qualifiera - peut-être à tort - de schizophrénique, donne lieu à un combat interne parfois intense entre les deux visages de ce moi dédoublé pour prendre le contrôle et imposer sa domination. Dans ces deux romans, est mis en scène l'effort du narrateur pour préserver son identité et son individualité face à une altérité logée dans son intériorité même, qui le rend pluriel et le place constamment sous l'épée de damoclès de la métamorphose, de la perte identitaire, de l'inexorable aliénation de soi. Si le traitement donné à cette thématique par les deux auteures laisse supposer la présence d'une pathologie jamais mentionnée, il est aisé d'imaginer que cette dualité qui tourmente Ana et Mina-Santiago est une dualité existentielle, universelle, la même qui affecte 
Jeckyll et Hyde, mais aussi et surtout le Ça et le Surmoi freudien de tout un chacun.

En effet, l'apport de la psychanalyse aux écritures du moi est indéniable: en brisant l'unité du sujet cartésien et kantien, unité qui était au cœur du projet autobiographique et qui en est même, selon Georges Gusdorf, le postulat ${ }^{1}$, les travaux de Freud ouvrent la voie à l'exploration des multiples dimensions du moi et de sa pluralité intrinsèque. Poussé à l'extrême et quelque peu dévoyé de son propos initial, ce constat de pluralité de l'individu pose la question de son inconstance, de son altérité essentielle et de la validité du concept d'identité, alors même que ce que pointent de plus en plus de récits sont les fragments épars qui composent ou décomposent l'individu.

C'est avant tout dans sa quête mémorielle que le sujet de l'introspection se révèle dans son incomplétude et sa fragmentarité. Le narrateur de La venganza de los pájaros de Guillermo Arreola cherche ses souvenirs d'enfance au fond d'une mémoire qui ne laisse affleurer que des bribes de son passé, soigneusement enveloppées d'un épais brouillard et recouvertes de cette même poussière ocre qui drappe tout le village familial. Les épisodes s'enchaînent, dans le désordre, au rythme des associations de pensées et en dépit de toute relation de chronologie ou de causalité. La démarche introspective, presque analytique, de ce monologue intérieur, n’offrira aucune révélation au narrateur devenu adulte, la convocation du passé ne suffisant pas à l'expliquer, à peine à l'exorciser. L'obsession de l'oubli, dont on ne sait pas s'il est une menace à éviter ou une promesse de libération vers laquelle tendre, parcourt le récit en même temps que les hallucinations oniriques facilitées par la lumière aveuglante qui les entoure. Le jeune homme, contrarié dans son récit par les souvenirs contradictoires des membres de sa famille, ne parvient pas plus que le lecteur à savoir ce qui relève de la mémoire ou de l'invention.

El Gran Vidrio (2007) de Mario Bellatin rassemble trois récits présentés comme trois autobiographies hautement improbables au cours desquelles les différents narrateurs plongent dans les territoires du rêve et du fantasme en brouillant délibérément les marqueurs d'authenticité et la dimension causale et chronologique de leurs histoires. Le jeune narrateur du premier chapitre navigue ainsi entre le récit de souvenirs immédiats ou plus anciens et le soupçon, cette fois-ci entièrement assumé par lui-même, que tout cela n'est que mensonge, que fantaisie. Là encore, le spectre de la folie n'est pas loin, puisque le garçonnet évoque un retard mental qui conduit à sa réclusion dans une institution spécialisée. Les problèmes mémoriels et de perception de la réalité ne sont pas les seules caractéristiques de ce narrateur chancelant, puisqu'au fur et à mesure du récit, dans les différents chapitres, sa voix finit par se mêler puis par se confondre avec celle d'un écrivain converti à l'islam soufi, puis d'une petite fille de quarante-

1 Georges Gusdorf analyse en ces termes les multiples obstacles qui se dressent entre l'objectif des écritures du moi et sa réalisation: «Les écritures du moi postulent l'unité et l'identité du moi, du commencement à la fin de son histoire.» (Gusdorf 1991: 304) 
six ans qui prend parfois l'aspect d'un adolescent aux lunettes carrées. La fièvre mythomane de l'invention de soi prend de l'ampleur, jusqu'à l'épilogue dans lequel toutes ces figures, tous ces moi réunis sous un je narrateur pluriel mais commun, déclarent leur fusion, leur équivalence, pour donner naissance à un narrateur mutant, hybride et multiple, qui se construit à grand renfort de fragments d'altérités authentiques ou inventées:

Mario Bellatin, el niño desnudo de grandes genitales, el personaje impresionado por la demolición de la casa familiar o el muchacho de lentes cuadrados que soñaba con tener una novia alemana. [...] Abdús Salam más bien, el hijo de la paz. [...] (Bellatin 2007: 164)

Cette pluralité identitaire se retrouve dans le cas tout aussi extrême de la Viajera del Vaso Vacío dont les lettres ponctuent le roman La muerte me da de Cristina Rivera Garza, et dans lesquelles elle adopte puis rejette successivement les identités de Lynn Hershmann, Gina Pane ou d'une Joachima Abramövic:

Me llamo Joachima Abramövic. Y no sé, en realidad, quién soy. [...]

Me llamaba Lynn Hershman, Pero todo muere, Cristina, ¿te acuerdas? Todo

cansa.

Ay Lynn, cómo te amé.

Mi nombre es Lynn. Lynn Hershman. Y mi nombre, como te lo imaginas, como lo sabes, como en tu propio caso, mi nombre no soy yo.

(Rivera Garza 2007: 79, 90, 92)

Si le moi apparaît fragmenté et pluriel, ses frontières sont également difficiles à définir: où s'arrête le je, où commence l'altérité? C'est un autre symptôme de l'écriture de l'introspection dans le roman mexicain récent. Les innombrables métamorphoses et travestissements des narrateurs de Mario Bellatin en constituent un exemple, mais c'est une dimension que l'on retrouve également en toile de fond de l'œuvre de Cristina Rivera Garza. Dans Verde Shanghai, deux narratrices s'imbriquent et se confondent par moments aux yeux d'une partie de leur entourage, mais aussi dans la tête de l'une d'elles, à la recherche d'un passé et d'une identité qui auraient été les siens. L'autre se débat pour échapper à cette femme qui la poursuit et représente tout ce qu'elle méprise le plus, et dont on ne sait plus si c'est une partie d'elle-même ou un alter ego qui lui ressemble à s'y méprendre. La muerte me da offre une autre variante de la porosité de la frontière entre le moi et l'autre: dans ce roman qui frise parfois le genre policier, trois personnages enquêtent sur des meurtres en série que rythment des poèmes d'Alejandra Pizarnik. Lintrigue se transforme rapidement en un parcours initiatique de recherche littéraire et métaphysique sui generis. On observe alors l'émergence d'une figure qui transcendera les limites de ce roman pour devenir une habituée du blog de l'auteure et l'héroïne de publications ultérieures: il s'agit de la Dama Pequeñísima. Cet être miniature naît dans la tête d'un des personnages et saute d'une conscience à une autre, devenant l'interlocuteur intime, l'alter ego imaginaire de l'un et l'autre. Cette projection hors de soi d'un bout de soi donne des airs de dialogues aux soliloques de ces personnages solitaires. Elle devient un instrument de choix de la démarche introspective, tendu comme un miroir 
de l'inconscient et comme un confortable substitut d'altérité, à mi-chemin entre l'étranger confesseur et l'alter ego condescendant et compréhensif.

Chacun à leur façon, ces narrateurs arborent tous une fracture, une faille qui laisse s'échapper un peu de leur cohérence subjective, en même temps que s'infiltrent des particules d'altérité et de folie, qui rendent périlleux l'accès à leur identité. Cette identité, ils parviennent parfois à l'entrevoir, elle prend la forme d'un mirage, d'une figure chancelante sur une corde raide au-dessus du gouffre vertigineux de l'aliénation, de lêtre-autre. Peut-être plus que la trame elle-même, ce vertige de soi affecte l'écriture jusqu'à la faire vaciller. Lorsque le sujet semble en déroute, les marqueurs langagiers s'affolent: chez Cristina Rivera Garza, les phrases agrammaticales, interrompues, saccadées, s'accumulent comme pour tenter de lutter contre le non-sens d'un langage fatalement inadéquat. C'est alors une logorrhée délirante, très proche du monologue intérieur, qui ne recherche en fin de compte aucune communicabilité, comme lorsque la narratrice, Cristina, se remémore la découverte du premier cadavre:

-Es un cuerpo- dije o debí decir, balbucir apenas, para nadie o para mí que no podía creerlo, que me negaba a creer, que nunca creí. Los ojos abiertos, desmesuradamente. El llanto. Pocas veces el llanto. Esa invocación. Ese crudo rezo. Lo estaba observando. No había escapatoria o cura. No tenía nada adentro y, alrededor de mí, sólo estaba el cuerpo. Lo que creí decir. Una colección de ángulos imposibles. Una piel, la piel. Cosa sobre el asfalto. Rodilla. Hombro. Nariz. Algo roto. Algo desarticulado. Oreja. Pie. Sexo. Cosa roja y abierta. Un contexto. Un punto de ebullición. Algo deshecho. (Rivera Garza 2007: 16)

Dans cette accumulation fiévreuse, anxieuse, convulsive et compulsive, dans ces abymes qui entourent les mots isolés, s'entrechoquant entre eux sans parvenir à se lier, se dessinent les lignes de fuite du langage et du sujet, en pleine dispersion. Dans El Gran Vidrio de Bellatin ou La venganza de los pájaros de Guillermo Arreola, les fragments de récits éparpillés sur les pages blanches, entourés de silence, laissent au narrateur le temps de reprendre son souffle, alors que les phrases numérotées et renvoyées à la ligne évoquent un halètement, un balbutiement, un spasme entre deux sanglots ou deux éclats de rire:

1. Durante el tiempo que viví junto a mi madre nunca se me ocurrió que acomodar mis genitales en su presencia pudiera tener una repercusión mayor.

2. Estaba equivocado.

3. Después supe que incluso les pedía a las otras mujeres objetos de valor para que los miraran plenamente.

4. Ajustados, acogotados, a punto de estallar.

5. Mi madre aprovechando mi dolor.

6. Recolectando objetos sin parar. (Bellatin 2007: 9)

Plus que cela, tous ces blancs du texte sont autant de constats de l'indicible, de l'inexprimable. C'est peut-être dans ces espaces dédiés au non-dit que s'imprime en négatif une part de la vérité subjective dans le récit.

Par ailleurs, les textes font état de l'incapacité du langage pour trouver et dire la vérité du sujet, et de l'obsolescence de la conception d'une vérité, unique et 
définitive: «Es difícil explicar lo que uno hace. Las causas. Las consecuencias. El proceso. Es difícil explicar lo que uno hace sin echarse a reír o a llorar, desmesuradamente.» (Rivera Garza 2007: 20), déplore Cristina, alors que Mario Bellatin s'exclame dans El Gran Vidrio: « ¿Qué hay de verdad y qué de mentira en cada una de las tres autobiografías? Saberlo carece totalmente de importancia.» (Bellatin 2007: 159). Les métamorphoses du narrateur de El Gran Vidrio, tout comme la multiplication des périphrases pour désigner un seul personnage, dans les romans de Cristina Rivera Garza, remettent également en question la permanence de l'identité. Selon les circonstances, la narratrice s'appellera «Cristina», «la Informante», «la Testigo» ou «la Sospechosa Inicial». Peutêtre encore plus symptomatiques sont les appellations de l'amant de celle-ci, alternativement «el Amante de la Gran Sonrisa Iluminada» et «El Hombre Que Era Él A Veces». Ces changements d'identité, mais aussi les fantômes qui peuplent l'univers du roman de Guillermo Arreola, s'opposent à toute exigence de vraisemblance, fondement même du pacte autobiographique qui, depuis Rousseau, fait de la vérité la condition sine qua non du genre. Dès lors que l’onirisme et la fantaisie la plus improbable émergent dans les nouvelles modalités de l'écriture introspective, le pacte en question se dilue dans la fiction; les carcans normatifs sétiolent à mesure que les écrivains prennent le contre-pied des modèles canoniques pour un renouveau joyeux et créatif du genre.

Jusqu'à leur progressive remise en question au cours de la seconde moitié du $\mathrm{xx}^{\mathrm{e}}$ siècle et de façon presque systématique ces dernières décennies, l'authenticité et la référentialité constituaient en effet le principal engagement des genres d'écriture de soi; même le roman à la première personne, lorsqu'il prenait des allures mémorielles, épousait l'apparence référentielle de l'autobiographie, il en revêtait le masque. Dans les œuvres qui nous intéressent, quelles fassent ou non état de prétentions autobiographiques, la réalité est sans cesse menacée par la fiction, la vérité par le mensonge, la lucidité par le délire. Quand le garçon de El Gran Vidrio de Bellatin craint de ne pas avoir toute sa raison, ou plus tard quand le narrateur ment et dément son autoportrait, c'est à la fois la crédibilité du narrateur et la frontière entre le rêve et la réalité qui s'effritent. Le protagoniste du dernier récit avoue volontiers: "Creo que soy algo mentirosa.» (Bellatin 2007: 135) et "Una de las características principales de mi personalidad es mentir todo el tiempo.» (Bellatin 2007 : 146). Il assume également l'épilogue dans lequel les narrateurs des trois chapitres se confondent finalement en une figure mouvante et plurielle, caméléon dont les métamorphoses se font au gré de ses caprices. Dans ce roman sous-titré Tres autobiografías, le pacte autobiographique de référentialité est troqué pour un nouveau pacte de fiction, qui n'exclut pas l'intervention d'éléments extratextuels mais propose d'abolir l'antagonisme traditionnel entre la fiction et la réalité. Tout se résout en un compromis de cohabitation ludique au service de l'invention littéraire, pour laquelle la quête d'une vérité illusoire ne présente plus guère d'intérêt.

En déployant les recours de la fiction autour de sujets aux prises avec le délire ou l'aliénation, ces auteurs s'inscrivent, avec ces écrits fictionnels ou 
autofictionnels, dans les inquiétudes propres aux sociétés occidentales de ce début de siècle, exacerbées par ce que Touraine appelle la crise de la modernité. Ce sont des personnages fêlés et inconstants qui se cherchent souvent en vain au détour de ces pages et s'exposent dans la nudité de leur imaginaire. Lobsession de la vérité factuelle fait place à l'intérêt pour les territoires de l'onirisme et $\mathrm{du}$ mensonge, dont on juge désormais qu'ils sont tout aussi authentiques ou mensongers que les données issues de la réalité référentielle extratextuelle, toujours soumise au soupçon de la déformation subjective. C'est peut-être en n'essayant pas de montrer la cohérence du sujet, mais au contraire en le dévoilant dans toutes ses inconsistances que cette génération s'approche au plus près de l'essence de la démarche introspective: l'impossible coïncidence de soi à soi.

La crise d'une époque et la crise du sujet entraînent dans leur sillage les genres référentiels d'écriture du moi et les discours qui leur étaient associés. Le langage est soumis au doute ambiant, on le surprend à tituber et à hoqueter ou à tourner en rond. Un nouveau sentiment d'absurdité existentielle s'empare des différents locuteurs introspectifs. Pour autant, le constat dressé nest pas celui de la stérilité littéraire ou esthétique, mais au contraire d'une forme de toute-puissance encouragée par la liberté avec laquelle on s'affranchit des normes pour donner naissance à un discours ludique, onirique et subversif capable de renouveler les modalités de l'écriture de soi et en même temps d'en saborder les piliers et les certitudes. Ces nouvelles poétiques de l'introspection sélèvent comme un chant métis et jouissif dans le paysage du roman mexicain contemporain. Bien loin de s'arrêter à la mort du sujet ou de l'auteur, elles signent au contraire un retour fracassant de celui-ci, qui s'arroge presque les pleins pouvoirs sur sa matière première, c'est-à-dire le langage et le récit, dévoyant l'un et l'autre au service de la fantaisie de ces fictions de l'intime.

\section{Bibliographie}

Arreola, G., 2006, La venganza de los pájaros, Mexico, Fondo de Cultura Económica.

Bellatin, M., 2007, El Gran Vidrio, Barcelone, Anagrama.

Gusdorf, G., 1991, Lignes de vie 1. Les écritures du moi, Paris, Odile Jacob.

Laurent Kullick, P., 2003, El camino de Santiago, Mexico, Biblioteca Era.

Lejeune, P., 1975, Le pacte autobiographique, Paris, Éditions du Seuil.

Menton, S., 1993, La nueva novela histórica de la América Latina, 1979-1992,

México, Fondo de Cultura Económica.

Nettel, G., 2006, El huésped, Barcelone, Anagrama.

Rivera Garza, C., 2007, La muerte me da, Mexico, Tusquets Editores.

Rivera Garza, C., 2011, Verde Shanghai, Mexico, Tusquets Editores.

Robbe-Grillet, A., 1984, Le miroir qui revient, Paris, Les Éditions de Minuit.

Sarraute, N., 1956, L’ère du soupçon, Paris, Gallimard.

Touraine, A., 1992, Critique de la modernité, Paris, Librairie Arthème Fayard.

Vargas Llosa, M., 2007, "Cien años de soledad. Realidad total, novela total», Cuadernos Hispanoamericanos, 681/2007, p. 9-12. 